\title{
Monitoring and Biochemical Treatment of Wastewater
}

\author{
Alina Dychko ${ }^{1 *}$, Natalya Remez ${ }^{1}$, Volodymyr Kyselov², Serhii Kraychuk², \\ Nataliia Ostapchuk ${ }^{3}$, Anna Kniazevych ${ }^{4}$
}

1 Institute of Energy Saving and Energy Management, National Technical University of Ukraine "Igor Sikorsky Kyiv Polytechnic Institute", 37 Peremohy Ave., 03056, Kyiv, Ukraine

2 Taurida National V.I. Vernadsky University, 33 Ivana Kudri Str., 04000, Kyiv, Ukraine

3 Rivne State University of Humanities, 12 Stepana Bandery Str., Rivne, 33000, Ukraine

${ }^{4}$ Stepan Demianchuk International University of Economics and Humanities, 4 Stepana Demianchuka Str., 33027, Rivne, Ukraine

* Corresponding author's e-mail: aodi@ukr.net

\begin{abstract}
The present paper provides the methodology for the environmental monitoring of natural and engineering wastewater systems, which involves the determination of the dichotomous fractal structure of the measuring network, the boundaries of the range and the density of pollution on the Peano and Koch curves, based on the data of the measuring network and the corresponding interpolation and smoothing algorithms, as well as determination of the dynamics of the pollution range using the Bayesian theorem. On the basis of the theory of fractals and the theory of sets, the developed algorithms for monitoring allow determining the structure of the measuring network taking into account the features of the controlled range and the sets of fractal isolines of any configuration with a given accuracy of reflection, which allows predicting the change in the composition of the effluent that comes to the reservoirs from the landscapes and improving the functioning of the equipment and environmental safety of water in general. The established dependence of biogas productivity on the different methods for destruction of the active sludge microorganisms allows determining that the maximum output of biogas occurs when applying the chemical destruction of part of the sludge.
\end{abstract}

Keywords: biochemical wastewater treatment, biogas, fractals, monitoring, reliability

\section{INTRODUCTION}

The problems with the lack of a systematic approach to the analysis pertaining to the functioning of natural and engineered sewage system and deficiency of accurate and operational measurements of qualitative and quantitative indicators characterizing the state of the system of drainage and water treatment are typical for the modern technologies of pollutants biotransformation during the wastewater treatment. Ensuring the data authenticity of the treatment process evaluation is an urgent problem, because a decision regarding the prediction of the treatment facilities operation and necessary measures for the protection of surface water bodies and the environment as a whole in the presence of the risk of natural or man-made accidents should be made on the basis of these data.

Therefore, the existing facilities for the biochemical wastewater treatment are characterized by insufficient purification efficiency due to the increasing quantity, concentration and variety of the environmentally hazardous pollutants; formation of significant volumes of sediments; consumption of large amount of energy and finances during exploitation; considerable time spent on treatment; need of significant territories under constructions, etc.

The vast majority of the methods for increasing the efficiency of the wastewater treatment process require additional stages, and 
consequently the construction of new treatment facilities, increased costs of material, energy, financial and other resources, which has no perspectives under the current economic conditions. Therefore, it is expedient to research and both improve the existing technologies in which the methods influencing the activated sludge are used in order to improve its functional and technological characteristics as well as substantiate the dependence of the effectiveness of the biochemical wastewater treatment on the influence of the biologically active substances of destructed sludge microorganisms [Yeremeev et al. 2012; Klymenko et al. 2013; Sean 2014].

The solution for the problem of wastewater treatment intensification should be based on the development of the innovative methods suitable for implementation at the existing treatment plants, which include the technological measures to improve the efficiency of the biotransformation process of pollutants and the organizational measures of process management, including effective monitoring of the drainage system state [Anpilova et al. 2007; Brack et al. 2017].

The development of a system for the environmental monitoring of the natural and engineered wastewater systems, as well as improvement of the existing environmentally safe technological processes and equipment for the biotransformation of pollutants ensures the rational use of water resources, compliance with the norms of effects of wastewater pollutants on the environment etc.

Proceeding from the above, the research and scientific substantiation of the principles for increasing the level of environmental safety of the biochemical wastewater treatment technology with the implementation of effective monitoring of pollutants is a relevant scientific and practical problem.

The idea of the research is the use of the scientifically-grounded measures to increase the level of environmental safety by ensuring the reliability of the monitoring data of the natural and engineered wastewater systems and intensification of wastewater pollutants biotransformation.

The aim of the research was to establish the regularities characterizing the biotransformation of wastewater pollutants with disintegrated biomass, aimed at increasing the level of environmental safety with monitoring of the natural and engineered wastewater systems.

\section{MATERIALS AND METHODS}

Ensuring the reliability of data monitoring of the natural and engineered wastewater systems is an urgent problem, because on the basis of these data, a decision on forecasting the development of enterprises and regions, the necessary measures for the protection of the environment and the population in case of the natural or man-made accidents should be made. The existing methods for increasing the reliability of the monitoring data are mainly based on the use of structural and procedural redundancy [Anpilova et al. 2007; Drigulich et al. 2007; Klymenko et al. 2013]. However, these methods are usually implemented selectively and unsystematically, which does not ensure compliance of the data with the requirements of the environmental standards. At the same time, more and more factors affect the state of sewage systems, most of which are very dangerous for natural reservoirs.

The natural and engineered drainage systems are the complex systems which are influenced both by the internal (technical, social, political, economic and environmental) and external (socio-political conflicts, global environmental problems, external debt, structural limitations) factors. Therefore, it is important to consider them in the context of the complex systems that can evolve.

The main reasons for the deterioration of the wastewater treatment quality include high concentration of pollutants in wastewater, sharp fluctuations of the quantitative indicators of drains quality, suboptimal content of biogenic elements in a culture fluid and others. In addition, outdated treatment equipment is not able to cope with the constant growth of certain chemical compounds in wastewater, such as phosphates etc.

In the scientific and technical literature, the violation of the biochemical wastewater treatment process is considered from the standpoint of one, two-, or several-factor analysis [Contreras et al. 2001; Sin et al. 2003]. The reasons for the low efficiency of the process either in terms of the design decisions, or physical parameters (temperature, speed of the stream, saturation with air) or chemical indicators (COD, concentration of substances) or biological characteristics of active sludge are emphasized. Thus, the researched methods of increasing the efficiency of treatment plants consider only one or two factors that affect the purification process. Practically, the methods of using disintegrated active sludge under anaerobic 
conditions are unexplored. In addition, there is no methodology for making decisions on the possible implementation of the intensification technologies taking into account the system of process factors.

The main ways of ensuring the quality of biochemical wastewater treatment is to intensify the process by adjusting the values of the main parameters; preliminary preparation or treatment of wastewater; structural changes in bioreactors; use of coagulants, flocculants, sorbents and other activating substances; the influence on microorganisms of active silt of magnetic, electric fields and ultrasound; chemical mutagenesis, etc. Intensification of the biochemical treatment contributes to a more efficient removal of organic matter, expanding the range of contaminants to be removed, to improve the sedimentation properties of the active sludge and to reduce its excess. In addition to the environmental benefits, the aforementioned methods also have economic advantages, which consist in reducing the capital costs for the construction of the wastewater treatment facilities and reducing the area occupied by plant buildings, reducing the electricity costs, increasing the oxidation capacity of the existing reactors without significant costs for their reconstruction, reducing the time for treatment, substantially increasing the reliability and stability of treatment at increased concentration of pollutants, no need for extra cleaning etc.

The essence of the methods for the biochemical treatment intensification by influencing the active sludge is that a part of the biomass is subjected to destruction, while its growth is decreasing, and also the intensity of processes increases significantly due to the release of enzymes, vitamins, proteins, polysaccharides and other biologically active substances that contribute to the stimulation of active sludge activity and, thus, the processes of biodegradation of complex organic pollutants present in wastewater in high concentration. The most effective methods of biochemical wastewater treatment intensification are thermal and chemical disintegration of active sludge cells [Sheng-bing et al. 2003].

\section{RESULTS AND DISCUSSION}

\section{Reliability of monitoring of natural and engineering wastewater systems}

The wastewater treatment enterprise management during the implementation of the intensification technology methods is associated with the following difficulties:

- it is necessary to take into account the system of parameters (up to several dozen) characterizing the process;

- there is no analytical dependence between the parameters of the process state and the reason for the efficiency reduction of the process. Determination of this dependence is complicated by a large number of state parameters and the diversity of their characteristics: quantitative, qualitative, binary, etc.;

- there are no databases of state parameters of the treatment facilities with the information on the verified reasons for reducing efficiency of treatment and the database of the process characteristics for wastewater treatment intensification, which can identify the purification process.

As a result of our own research, the methodology for the monitoring of the natural and engineered wastewater systems was developed. The monitoring of the wastewater treatment systems includes the stages of determining the current state of the existing pollutants, concomitant factors and their impact on the spectrum and magnitude of pollution, the dynamics of pollution in comparison with previous measurements, checking the conformity of contamination trends of pollution, which had taken place before, and also making a decision about the reliability of the data monitoring and forecasts.

The assessment of the state of the natural and engineered wastewater systems includes the development of a measuring network by presenting it as a Peano curve [McVay et al. 2006], ensuring a continuous monitoring of the researched system and the absence of gaps between individual points of observation. After determining the structure of the measuring network, the volume of the samples (measurements) is optimized in order to exclude the possible influence of random obstacles.

During the formation of the fractal structure of pollution range, it is necessary to consider that there is a rigid dependence of the height of the equilateral approximating triangle on the length of the segment of approximation $L$. This forces choosing such length of the initial interval of approximation $L_{0}$ that the amplitudes of the maximum deviations of the real values of the actual distribution $\left.F^{R}\right)(f)$ from the approximating line segment on this segment are compatible with the height of the triangle $h_{10}=\left(L_{0} / 3\right) \sin 60^{\circ}=0.288 L_{0}$. 
In order to eliminate this disadvantage, a modified fractalization algorithm, which involves the creation of a dichotomous fractal structure, is proposed to use [Chung et al. 2006]. The result of fractalization (a model that approximates the "chaotic" process on the segment of approximation) can be written as the fractal value $L_{m}=L_{0}\left(2^{m-1}\right)^{-1}$, where $m$ - number of steps performed during the construction of the fractal structure, as well as a set of $2^{m-1}$ values of fractal coordinates.

The next step is the use of the methods of hybrid monitoring of the system state, which consists in choosing from a plurality of relevant models the one for which the Euclidean distance is the smallest relatively to the real measurement data executed at the time specified by the model. If the metrics that characterizes the distance between the curve of the point distribution during real measurement $\left(f^{\prime}\right)$ and the curves obtained by modeling with the help of the model $m^{j}\left(f^{m j}\right)$ are considered, then the increment of information characterizing the degree of closeness $f\left(r^{\prime}\right)$ and $f\left({ }^{m j}\right)$, is as follows:

$$
d\left[f^{(r)}, f^{(m)}\right]=\left(\sum_{i=1}^{N}\left(x_{i}^{(m)}-x_{i}^{m}\right)\right)^{1 / 2}
$$

where: $N$ - number of points at which the measurements are made,

$X^{(r)}{ }_{i}$ and $X^{(m)}$ - respectively, value of actually determined by measuring and calculated using the model of pollution value at the $i$-th point.

At the same time, the smallest of the calculated metrics corresponds to the model that describes the best the process of pollution migration, that is, a model satisfying the following equation:

$$
\begin{aligned}
& \operatorname{opt~} \mathrm{d}_{\mathrm{j}}\left[\mathrm{f}^{(\mathrm{r})}, \mathrm{f}^{(\mathrm{mj})}\right]=\min \left\{\mathrm{d}_{2}\left[\mathrm{f}^{(\mathrm{r})}, \mathrm{f}^{(\mathrm{m} 1)}\right],\right. \\
& \left.\ldots, \mathrm{d}_{\mathrm{j}}\left[\mathrm{f}^{(\mathrm{r})}, \mathrm{f}^{(\mathrm{m} 2)}\right], \ldots, \mathrm{d}_{\mathrm{k}}\left[\mathrm{f}^{(\mathrm{r})}, \mathrm{f}^{(\mathrm{mk})}\right]\right\}
\end{aligned}
$$

This stage is completed by defining the number and nomenclature as well as the absolute values of pollution that were not previously observed in this area, the boundaries of critical pollution, the development of the equidistant contamination and the formation of the corresponding (source) file in facts bank.

The next stage of monitoring is an establishment of the correlations between the concomitant and middling factors and actual monitoring data, as well as the analysis of migration and metabolism of contaminants under specific conditions (taking into account such processes as transfer, gravity deposition, dissolution, deflation, chemical and biological processes, radioactive decay, etc.).

The metrics calculation describes the distance of the set of data for the current and previous measurement cycles as well as the change vector. Detection of the contamination dynamics ensures an early change in the methodology for the determination of pollutants and the replacement of the measuring equipment, which is associated with a change in the range of measurements and the possible decrease in the sensitivity of the instruments [Yeremeev I.S. et al. 2013; Yeremeev I.S. et al. 2019; Hafeez et al. 2018].

The task of identifying the dynamics of the pollution field is to analyze the observations and compare their results with each other by evaluating the metrics. The discrepancy in the case of comparing the results of the past ("reference") and modern observations is characterized by a metrics

$$
\mathrm{d}_{\mathrm{E}}\left(\mathrm{f}^{(1)}, \mathrm{f}^{(2)}\right)=\sqrt{\frac{1}{\mathrm{n}-1} \cdot \sum_{\mathrm{i}=1}^{\mathrm{n}}\left(\mathrm{x}_{\mathrm{i}}^{(2)}-\mathrm{x}_{\mathrm{i}}^{(1)}\right)^{2}}
$$

where $n$ - number of nodes in the measuring network.

The metrics estimates the degree of information gain of the system state in the distribution of $n$ elements of the set $f^{(2)}$ observed during the current control procedure in accordance with the information of the environment state that corresponds to the distribution $f^{(l)}$ that was evaluated during the previous control procedure and was adopted as a standard. Moreover, if the statistics $f^{(I)}$ is sufficiently well defined, the mathematical expectations are used instead of the $f^{(1)}$ elements. The information gain determined in the case of replacing $f^{(2)}$ by $f^{(1)}$ can be considered as nonessential, and the distributions themselves are identical only when the statistics $f^{(I)}$ absorbs $f^{(2)}$ statistics or when statistics data are absent, but the value of the measure does not exceed the double error inherent in the method used for the analysis of the samples.

In the opposite case, this gain characterizes the state of the dynamics during the period between observations. The incompleteness of the output data, which is the result of the limited possibilities of the controlled objects monitoring and insufficient information about the factors that influence the processes in the system, indicates that if

$$
\mathrm{V}(\mathrm{N}, \mathrm{f})=\{\widetilde{\mathrm{f}} \in \mathrm{F}: \mathrm{N}(\widetilde{\mathrm{f}})=\mathrm{N}(\mathrm{f})\}
$$


that is, the set of all elements that do not differ from the element $f$ using information $N$, then the value of $N(f)$ does not allow determining which of sets $S(\widetilde{\mathbf{f}}, \xi), \widetilde{\mathbf{f}} \in V(N, f)$ corresponds to the $\xi$-approximation that is desirable. In this case, additional information is needed and the necessary $\xi$-approximation can be found only under the condition that the intersection of the sets $(\xi)$ is not empty, that is

$$
\begin{aligned}
& \mathrm{A}(\mathrm{N}, \mathrm{f}, \xi)=\mathrm{MIN}\left\{\mathrm{S}(\mathrm{f}, \xi)^{1} 0\right\}, \\
& \widetilde{\mathrm{f}} \in \mathrm{V}(\mathrm{N}, \mathrm{f})
\end{aligned}
$$

or

$$
\begin{aligned}
& \mathrm{D}(\mathrm{N}, \mathrm{f}, \xi)=\mathrm{MIN}\{\mathrm{f}(1), \ldots \mathrm{f}(\mathrm{k})\}, \\
& \widetilde{\mathrm{f}} \in \mathrm{V}(\mathrm{N}, \mathrm{f}),
\end{aligned}
$$

where MIN - operator of multivalued logic corresponding to the logical multiplication operation.

Then, an analysis of the possible effects of external factors, which are presented in the monitoring data format as concomitant or middling factors, is provided. If the deviation of the trend is not significant, the external factors take place and are characterized by significant amplitude, an analysis of the reasons for the invariance of the monitoring results is necessary.

The final stage in assessing the state of the natural and engineered wastewater systems is to test the hypothesis of the monitoring results, the overall assessment of the reliability of the data, and the formulation of a decision on the accuracy of estimates and forecasts. The application of the Bayesian theorem here allows determining, and subsequently clarifying, the validity probability of the accepted data distribution hypothesis under the conditions of uncertainty that exists during monitoring. According to this method, the probability is calculated taking into account both the previously known and new obtained information. The validation results with a check mark are sent to the relevant file of facts bank to replace the corresponding output file.

The consistent implementation of the abovementioned stages of the evaluation of information ensures the reliability of the obtained data to make decisions in the environmental management of wastewater treatment plants.

Using the results of observations and analogies gives the reason to consider that the structures and functioning of natural and engineering drainage systems demonstrate self-similarity in a broad sense: water purification from pollution under both the natural conditions and in wastewater treatment facilities proceeds according to the same principles and differs only in terms of the productivity and quality of purification, i.e., systems more or less equally fit into a wide range of spatial, temporal and quantitative scales (measures), indicating the presence of certain symmetry scales. In this case of the research on such systems, the methods of fractal analysis and elements of the theory of chaos can be used [Potapov 2016].

For formation an adaptive development cycle (system model), it is necessary to take into account three main characteristics:

- inherent potential of the system, which allows making the necessary changes in case of their needs (resource);

- internal control (connectivity) of the system, that is, the degree of connectivity between internal controlled variables and processes (a measure that reflects the degree of flexibility or rigidity of such control, sensitivity or insensitivity to perturbations);

- the ability to adapt, the elasticity of the system (the extent of its vulnerability to unexpected or unpredictable stresses).

In order to provide the adaptation processes in the system, the heuristics can be formulated [Yeremeyev et al. 2019]. If the following abbreviations are accepted:

NM - new model; DVP - development; TNM - transition to a new model; ROR - reorganization; CR - crisis; AD - adaptation; GR gain of the resource; GC - gain of connectivity, $\mathrm{SC}$ - system conservation; LACR - local artificial crisis (reorganization of the local subsystem in order to adapt it to new conditions or requirements), VR - value of a risk in case of transition to LACR, then the functioning of the system and the corresponding corrective actions can be described as follows:

$$
\begin{aligned}
& \text { if }([\mathrm{GR}>0] \text { and }[\mathrm{GC}>0]) \text {, then }(\mathrm{DVP}) \text {; } \\
& \text { if }([\mathrm{GR} \sim 0] \text { and }[\mathrm{GC}>0]) \text {, then }(\mathrm{SC}) \text {; } \\
& \text { if }([\mathrm{GR}<0] \text { and }[\mathrm{GC}<0]) \text {, then }(\mathrm{CR}) \text {; } \\
& \text { if }([\mathrm{GR}>0] \text { and }[\mathrm{GC}<0]) \text {, then }(\mathrm{ROR}) \text {; } \\
& \text { if }([\mathrm{GR}<0] \text { and }[\mathrm{GC} \sim 0]) \text {, then }(\mathrm{AD}) \text {; } \\
& \text { if }([\mathrm{GR}<0] \text { and }[\mathrm{GC}>0] \text { and } \\
& \left.\left[\mathrm{VR} \leq \mathrm{VR}_{\mathrm{acc}}\right]\right) \text {, then }(\mathrm{LACR})
\end{aligned}
$$

where $V R a c c$ - maximum acceptable VR under these conditions.

Since functioning at each level is cyclical and as the initial conditions for each cycle are not usually the same, there are the conditions for creating 
"chaos", that is, unpredictable development of events that is typical of the biochemical purification systems.

In the case of the population development under the conditions of different initial data that are characteristic of the operation of treatment facilities, the expected density of the population of active sludge microorganisms at the time $t+1 N_{t+1}$ can be represented as a nonlinear autoregressive model:

$$
\mathrm{N}_{\mathrm{t}+1}=\mathrm{N}_{\mathrm{t}}\left[1+\breve{\mathrm{r}}\left(1-\mathrm{N}_{\mathrm{t}} / \mathrm{K}\right)\right],
$$

where $N_{t}$-density of the population at time

$t, \breve{r}$ - internal rate of population growth (Malthusian parameter), and

$K$ - the maximum volume of this population, possible for these conditions.

After certain transformations, this model can be represented as

$$
\mathrm{x}_{\mathrm{t}+1}=\mathrm{rx}_{\mathrm{t}}\left(1-\mathrm{x}_{\mathrm{t}}\right),
$$

where $x_{t}=N_{t} \check{r} /[(1+\check{r} K)]$, and

$r=1+\check{r}$, so that $x_{t}-$ dimensionless quantity that characterizes the population.

The presented model allows obtaining solutions in rather wide limits, which, on the one hand, do not allow uniquely predicting the result, and on the other hand-indicating the limits in the middle of which the system behaves in a certain way predictably, which allows assessing the reliability of data specific measurements.

Wastewater treatment is characterized by the use of averaged data associated with statistical errors such as the standard deviation $\sigma$, when within the double deviation of $2 \sigma$ there is uncertainty about the true value of a particular parameter, as well as the presence of so-called trust intervals.

In the case of the necessity to determine the cause-effect relationships and prediction of further development of events, it is necessary to apply an approach based on the estimation of the values of "nearest neighbors" (single-link method), which are within the accepted $2 \sigma$ limits. In order to assess this trend, the following heuristics can be used:

$$
\begin{aligned}
& \text { IF ([MSTAB] AND [SIGN } \left.S S T_{i \neq j} E Q\right] \text { ), } \\
& \text { THEN [CTO] }
\end{aligned}
$$$$
\text { where } M S T A B \text { - situation where the measured }
$$
value does not exceed the standard deviation,

$S I G N \Delta S T_{i \pm j} E Q$ - observation result which reveals that the sign of state changes at current measurement at all points to the left and right of $i$, that is, at points from $i$ to $i-j$ and from $i$ to $i+j$, the same relatively to the state at the same points during the preliminary measurement or in the spatial distribution,

CTO - certain tendency is observed.

Ensuring the quality of wastewater treatment should include: observation of the states and degrees of the possibility of the conditions; definition of states that are not possible at the additional information; predictions of states that are not observable but are fundamentally possible and which gives the nonzero degree of $f_{M}(c)$ that is less than the minimum degree of $f_{M}(\alpha)$ possibility, which is calculated for the observed states, for example

$$
\mathrm{f}_{\mathrm{M}}(c)=0.5 \underbrace{\min }_{\alpha} f_{M}(\alpha)
$$

$$
\begin{gathered}
\mathrm{D}_{\mathrm{p}}\left[f_{M}(c), f_{M}(\alpha)\right]= \\
=\left\{\sum_{c \in C}\left[f_{M}(c)-f_{M}(\alpha)\right]^{p}\right\}^{1 / p} \subseteq 0.5 \underbrace{\min }_{\alpha} f_{M}(\alpha),
\end{gathered}
$$

where $p$-parameter of the function of the distance $D p$ (for the Euclidean distance $p=2$ ).

The ability to predict the states that are not observed involves the presence of information from the outside of the system, or the information link between the event, the phenomenon and the state of the system. Such a connection is not unambiguous and allows evaluating the possible states of the system only at a qualitative level, moreover with an error.

Therefore, it is expedient to determine the increment of the Euclidean distance between the pairs of real states that are observed and are adjacent to each other in this time interval, as well as the probability and possibility of such a transition and the driving forces (internal and external) that contribute to this. Establishing the fundamentally possible states that cannot be recorded online, but which can globally affect the system and its indicators, allows them to assess their possibility and the subsequent consequences.

If the measure of confidence increase $M B$ is presented to the hypothesis $h$ based on the observation of the output $e$ as:

$$
\mathrm{MB}[\mathrm{h}, \mathrm{e}]=\{\mathrm{P}(\mathrm{h} \mid \mathrm{e})-\mathrm{P}(\mathrm{h})\} /(1-\mathrm{P}(\mathrm{h})),
$$

where $P(h / e)-$ conditional probability $h$ for the known $e$, and 
$P(h)$ - an expert estimation of the probability for a given moment of time, then the extent of the increasing $M D$ 's uncertainty with respect to the hypothesis $h$ can be represented as

$$
M D[h, e]=\{P(h)-P(h \mid e)\} / P(h),
$$

and the factor of uncertainty $C F$ :

$$
\mathrm{CF}[\mathrm{h}, \mathrm{e}]=\mathrm{MB}[\mathrm{h}, \mathrm{e}]-\mathrm{MD}\{\mathrm{h}, \mathrm{e}] \text {. }
$$

The obtained values $M B, M D$ and $C F$ for each case can be used to compile heuristics for system management under uncertain conditions. These heuristics allow improving the quality of the wastewater biochemical treatment procedures under uncertain conditions and the effects of factors that are poorly evaluated.

Each operating rule for choosing the method of intensifying the wastewater treatment can be presented in the form "IF" \{conditions\}, "THEN" \{consequences\}. Thus, the heuristics for the methods of the process intensification can be formulated as follows:

if [\{(VSS_A) and (BCO_A) and (CAS_A) and (IAS_A) $\}$,

or $\{($ VSS_S $)$ and (BCO_G) and (CAS_G) and (IAS_G) $\}]$, then $\{$ NIP_G $\}$, if $\left\{\left(\mathrm{VSS}_{-} \mathrm{A}\right)\right.$ and $\left(\mathrm{BCO} \_\overline{\mathrm{G}}\right)$ and

(CAS_G) and (IAS_A) $\}$, then $\{$ NIP_A $\}$, if $\left\{\left(V S S \_G\right)\right.$ and $\left(B C O \_A\right)$ and $\left(C A S \_A\right)$ and (IAS_G) $\}$, then $\left\{N I P \_\bar{G}\right\}$

where VSS - velocity of the wastewater stream, $C A S$ - concentration of active sludge, $I A S$ - index of active sludge (reflects its properties),

$N I P$ - necessity for intensification of treatment process, and $A, G, S$, - relevant estimates (average, great and small).

The practical realization of the conclusions of the theorems for identifying the state of the systems is accomplished by describing the behavior of the system by a set of alternative models, each - under certain difficult conditions - can be identified as the best. In these circumstances, one can turn to the expert estimate of the consequences of the coexistence of some indeterminate set $x^{(i)} \in X$, which is actually measured, the data on the state of controlled treatment system process with a number of standards $y^{(i)} \in Y$, followed by the consequences of state simulation of the same system with a number of alternative models. To do this, it is first of all necessary to determine the structure of the formed set of prototypes $Y$, which is characterized by a certain reference ratio. Then, the type of relation between two sets of $X$ and $Y$ and the meanings of its evaluation are chosen. Finally, the relationship researched with the reference relationship is determined, and a decisive rule about the state of the systems is formed.

\section{Efficiency of biogas output from wastewater treatment}

In order to establish the regularities of the biotransformation of wastewater pollutants with disintegrated biomass, an experimental research with aerobic and anaerobic wastewater treatment with using destructive methods of active sludge was carried out.

The ways which promote maximization of energy consumption and, at the same time, give a high purification effect for wastewater treatment are presented in Fig.1.

Thus, the highest purification efficiency for COD is achieved by applying mechanical degradation and adding part of the dried active sludge to the bioreactor. The best removal of nitrogen and phosphates from wastewater occurs when applying the chemical treatment of biomass.

It has been experimentally proven that the most effective methods for the destruction of the odd active sludge for its use in biochemical wastewater treatment are mechanical and chemical disintegration, at which there is an increase in the degree of wastewater treatment. At the mechanical treatment of part of active sludge, the removal of pollutants from wastewater is the most intensive. However, the concentration of nitrogen in the wastewater purified during the chemical treatment of sludge does not exceed the allowed concentration, in contrast to the mechanical one. Destruction of active sludge as a result of its treatment with thermolysis at a temperature of $90^{\circ} \mathrm{C}$ leads to a decrease in its growth, but it has the smallest, compared with other methods, effect of the COD removal (77\% vs. $90 \ldots 99 \%)$.

The maximum purification efficiency - up to $99 \%$ is achieved with use of disintegrated biomass under anaerobic conditions by adding a portion of the active sludge treated with hydrogen peroxide to the methantank (Fig. 2).

Significant degree of wastewater purification by COD is observed in control and with mechanical destruction - up to $93 \%$. When processing the 


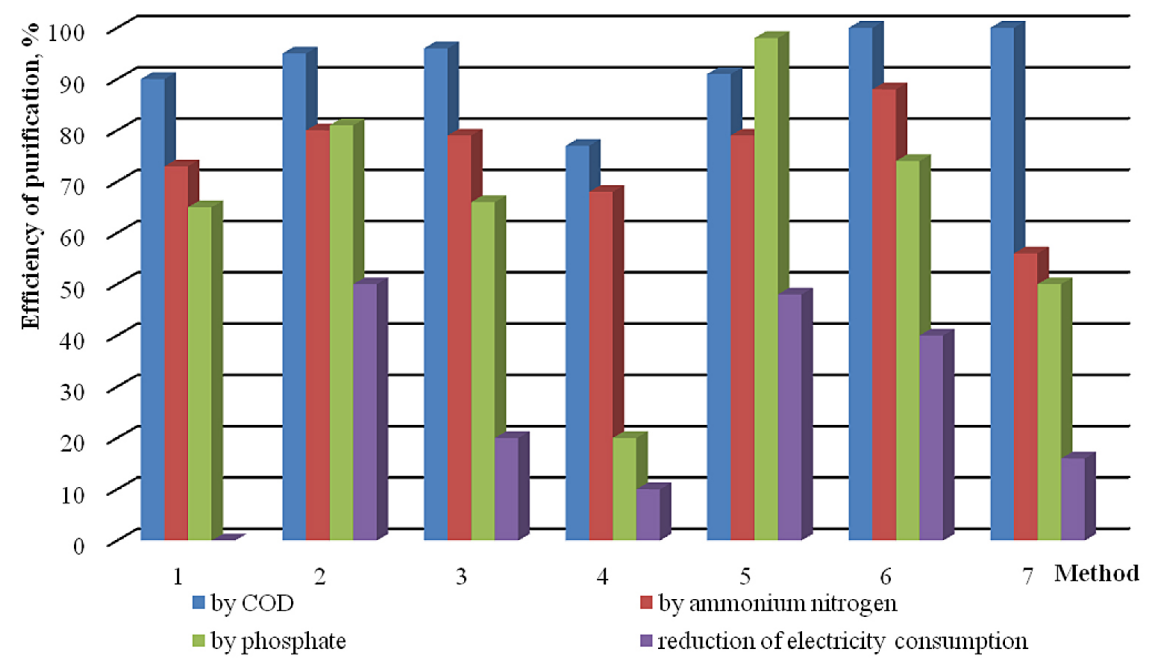

Fig. 1. Efficiency of wastewater purification and reduction of energy consumption during intensification of the process: 1 - control wastewater; process with use of: 2 - chemical destruction; 3 - mechanical destruction; 4 - thermolysis; 5 - heating; 6 - dried sludge ( $1 \%$ of the total volume of construction); 7 - dried sludge ( $3 \%$ of the total volume).

biomass part of the active sludge with the method of thermolysis, the purification rate is reduced to $85 \%$ compared with other methods.

The most intensive formation of biogas is observed during the mechanical and chemical degradation of a part of the biomass - up to 7 and 10 liters / liters (1 biogas / 1 of wastewater), respectively (see Fig. 2).

As a result of the destruction of active sludge cells, the impurities of biological origin, humus substances, molecular organic substances, phosphates and others are removed from wastewater more intensively. In addition, under the influence of hydrogen peroxide, the speed of the overwhelming majority of the oxidative-reducing chemical reactions increases, which also leads to the intensification of biotransformation of pollutants in wastewater.

As a result of research and its analysis, the practical measures and recommendations for ensuring the quality of biochemical wastewater treatment were developed based on the application of the most effective methods of intensifying the process, taking into account such principles of analysis of complex natural and engineering wastewater systems as: simplification of processes and structures for their adequate understanding; considering them in dynamics and prospects; taking into account the possibility of ambiguity and unpredictability; understanding of the system as a hierarchical structure suitable for adapting and developing.
On the basis of the research, the technological scheme of intensification of the wastewater treatment process was substantiated, which differs from regeneration of active sludge included in the biochemical purification scheme, consisting in the processing of the part of biomass of the active sludge by the method of physical or chemical destruction. The developed technology for intensification of pollutant biotransformation in the anaerobic wastewater treatment includes a node of destruction of the odd active sludge, namely physical or chemical destruction of biomass after stage I of methane fermentation with its referral to the II stage, which improves the quality of treated wastewater, allows obtaining an additional source of energy, i.e. biogas with higher concentrations of methane, and increasing the environmental safety of natural waters and the environment as a whole [Dychko et al. 2018].

The economic effect after the implementation of the active sludge destruction in the process of biochemical wastewater treatment is from 35 to 1000 TEUR, which is $10-17$ times more than traditional anaerobic technologies . Such a high effect from the implementation of the developed technology is associated with an increase in the biogas output and increased methane content in it, which significantly affects the energy intensity of biogas. The payback period of the developed technology is $1.5-2.6$ years. 


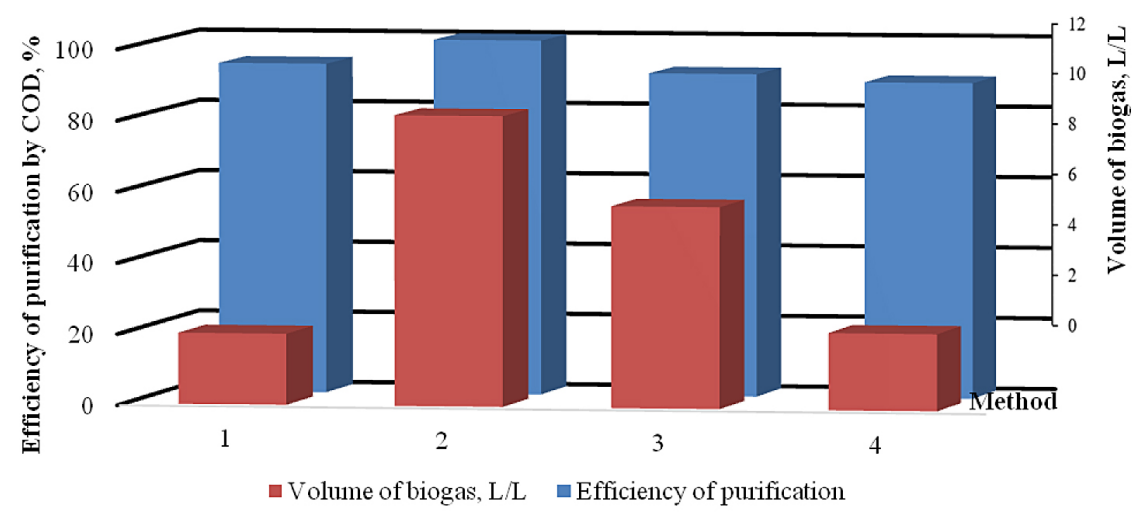

Fig. 2. Efficiency of wastewater purification and biogas output during process intensification under anaerobic conditions: 1 - control wastewater; with use of: 2 - chemical disintegration, 3 - mechanical disintegration, 4 - thermolysis.

\section{CONCLUSIONS}

1. The developed methodology for increasing the data reliability of environmental monitoring of the natural and engineered wastewater systems involves the determination of the dichotomous fractal structure of the measuring network, the boundaries of the range and the density of pollution with the Peano and Koch curves, based on the data of the measuring network and the corresponding interpolation and smoothing algorithms, as well as determination of dynamics of the range pollution using the Bayesian theorem.

2. On the basis of the theory of fractals and the theory of sets, the algorithms of monitoring of the natural and engineered wastewater systems allow determining the structure of the measuring network taking into account the features of the controlled range and the sets of fractal isolines of any configuration with a given accuracy of reflection; this allows predicting the change in the composition of the effluent that comes to the reservoirs from the landscapes, as well as in case of floods and other disasters, and improving the functioning of the equipment and environmental safety of surface water in general.

3. It was established that the most significant factors in the biochemical purification of wastewater are the oxidizing capacity of the treatment facilities, the temperature of the process and the presence of nutrients in the equipment.

4. It was established that during the destruction of active sludge by hydrogen peroxide, the most effective biotransformation of pollutants occurs, achieving the degree of wastewater treatment up to $99 \%$ for COD (up to $5-10 \mathrm{mgO}_{2} / 1$ ), the removal of nitrogen-containing and phosphorus-containing organic compounds - up to $90 \%$ (up to 7 and $3 \mathrm{mg} / 1$ respectively), reducing the amount of excess biomass production up to 8 times, and also obtaining biogas, the amount of which in 4-5 times greater than in the methantank operating with the nontreated biomass.

5. The established dependence of the biogas productivity with different methods of destruction of active sludge microorganisms allowed determining that the maximum output of biogas - up to $10 \mathrm{~m}^{3} / \mathrm{m}^{3}$ of wastewater - takes place when applying the chemical destruction of part of the sludge.

\section{REFERENCES}

1. Anpilova E.S., Voloshkina O.S., Trofimchuk O.M. et al. 2007. Use of GIS technologies for inventory of sources of pollution of surface waters of transboundary objects. Ecology and resources. 16, 46-51 (in Ukrainian).

2. Brack W., Dulio V., Ågerstrand M. et al. 2017. Towards the review of the European Union Water Framework Directive: Recommendations for more efficient assessment and management of chemical contamination in European surface water resources. Science of the Total Environment. 576, 720-737.

3. Chung K., Hsu C. 2006. Novel prediction- and subblock-based algorithm for fractal image compression. Chaos, Solitons \& Fractals. 29 (1), 215-222.

4. Contreras E., Bertola N.\& Zaritzky N. 2001. The application of different techniques to determine activated sludge kinetics parameters in a food industry wastewater. Water SA. 27 (2), 34-36.

5. Drigulich P.G. \& Kalinkin O.G. 2007. Methods of 
monitoring the state of the environment in the field of drilling waste disposal (on example of Andryushivsky deposit). Oil and gas industry. 4, 51-54 (in Ukrainian).

6. Dychko A., Remez N., Opolinskyi I. et al. 2018. Modelling of Two-Stage Methane Digestion With Pretreatment of Biomass. Latvian Journal of Physics and Technical Sciences. 55 (2), 37-44.

7. Hafeez S., Wong M., Abbas S. et al. 2018. Detection and Monitoring of Marine Pollution Using Remote Sensing Technologies. Monitoring of Marine Pollution, Houma Bachari Fouzia, IntechOpen. DOI: 10.5772/intechopen.81657.

8. Klymenko M.O., Klymenko O.M. \& Petruk A.M. 2013. Hydroecological monitoring of water ecosystems in view of modern European directions in environmental protection activities. Bulletin of Poltava State Agrarian Academy, 3, 22-27 (in Ukrainian).

9. McVay J., Hoorfar A. \& Engheta N. 2006. Theory and experiments on Peano and Hilbert curve RFID tags. Proceedings of SPIE - The International Society for Optical Engineering. 6248. 10.1117/12.666911.
10. Potapov A. 2016. Chaos Theory, Fractals and Scaling in the Radar: A Look from 2015. 0.1007/978-3-319-29701-9 12.

11. Sean L.X. 2014. Food and Agricultural wastewater utilization. Rutgart: Blackwell publishing.

12. Sheng-bing H., Bao-zhen W., Lin W. et al. 2003. Determination of operational parameters of anaerobic phase for enhanced phosphorus removal in MBR. Journal of Environmental Sci. 15 (6), 749-756.

13. Sin G., Malisse K. \& Vanrolleghem P.A. 2003. An integrated sensor for the monitoring of aerobic and anoxic activated sludge activities in biological nitrogen removal plants. Water Science and Technology. 47 (2), 10-12.

14. Yeremeev I.S. \& Dychko A.O. 2012. Management of quality of biochemical wastewater treatment. System Research \& Information Technologies, 4, 45-48 (in Ukrainian).

15. Yeremeyev I., Dychko A., Kyselov V. et al. 2019. Model Monitoring and Evaluation of Radioactive Contamination. Latvian Journal of Physics and Technical Sciences. 56 (4), 57-67. 